\title{
Efficient photochemical generation of peroxycarboxylic nitric anhydrides with ultraviolet light-emitting diodes
}

\author{
N. D. Rider et al. \\ Correspondence to: H. D. Osthoff (hosthoff@ucalgary.ca)
}

The copyright of individual parts of the supplement might differ from the CC-BY 3.0 licence. 


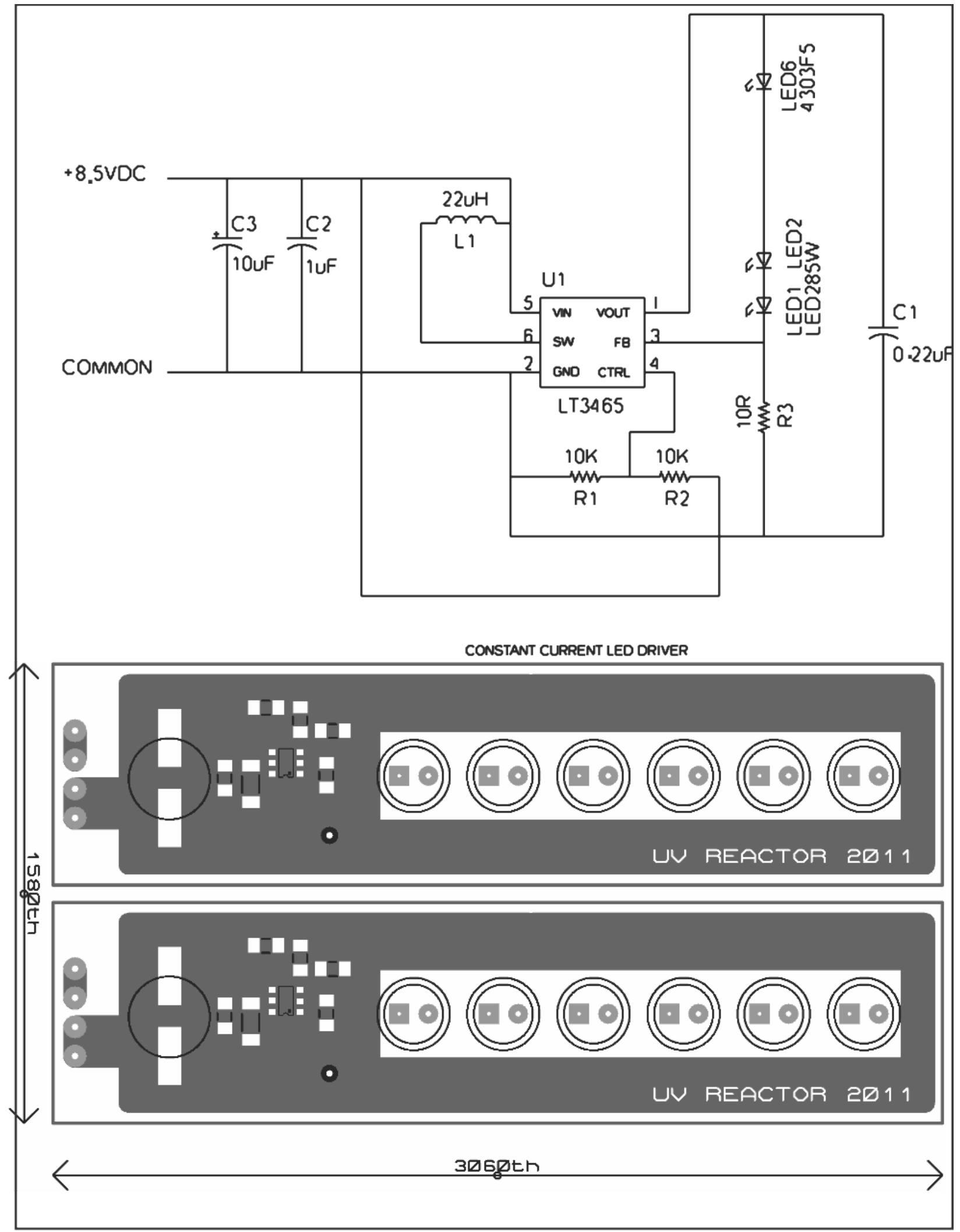

Figure S1. Electronic schematic of the UV-LED driver. 


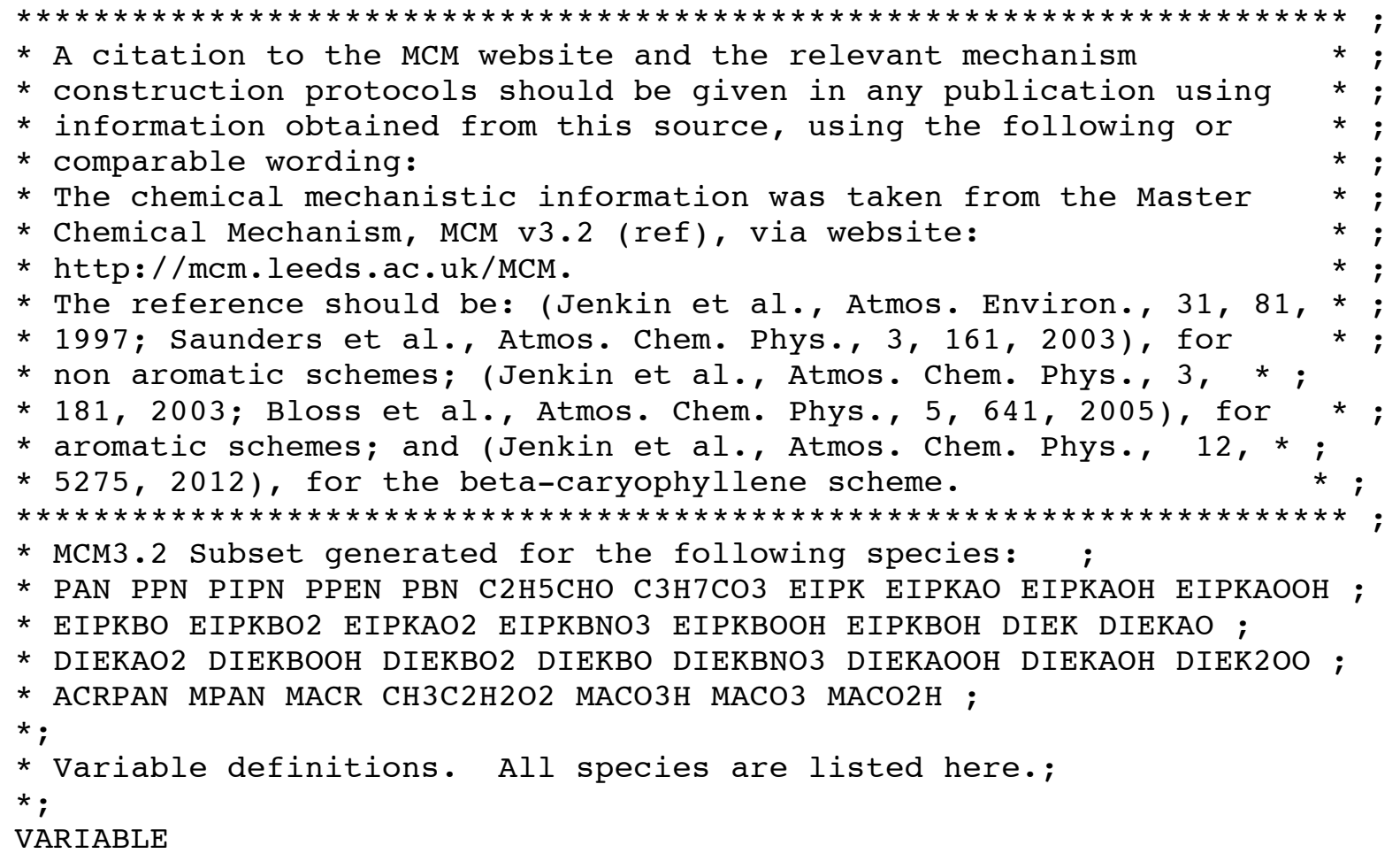

C4PAN 1 HO1C3O C4PAN4 HO2C3CO3 MEKCO CH3C2H2O2 ETHENO3O BUTALO2 $\mathrm{CH} 3 \mathrm{NO} 3 \mathrm{C} 2 \mathrm{H} 5 \mathrm{O} 2 \mathrm{NO} 3 \mathrm{CH} 2 \mathrm{CO} 2 \mathrm{H} \mathrm{C} 23 \mathrm{C} 54 \mathrm{CO} 3 \mathrm{MEKAOH} \mathrm{CO} 3 \mathrm{C} 4 \mathrm{CO} 2 \mathrm{H}$ DIEKAOH BIACETO2 HOC2 4 CO2H DIEKBNO3 HO1CO3C5 MACROHO2 O3 PAN CH3COCH2O IPROPOL PERBUACID C6PAN7 HYPROPO ETHGLY EIPKAOOH MACROH C66OH HOCO3C54O $\mathrm{HCOCH} 2 \mathrm{CO} 3 \mathrm{MEKCOOH}$ NBUTOLAO2 $\mathrm{HCOCO} 3 \mathrm{H} \mathrm{C} 5 \mathrm{CO} 234 \mathrm{C} 2 \mathrm{H} 5 \mathrm{CO} 3 \mathrm{HCOCO} 3 \mathrm{NC} 4 \mathrm{H} 9 \mathrm{NO} 3$ HSO3 GLYPAN HCOCH $2 \mathrm{O} 2$ NC3H7OOH CH3O NBUTOLAOH CO3C54CHO NBUTOLAOOH IPROPOLPAN IBUDIALCO3 MACRO2 IC3H7NO3 BUTALO ACO3H PROPALOOH IPRHOCO2H ETHOHNO3 ACO2H PPEN OH NBUTOL BIACETOH DIEKAO2 HOIPRCO3 PROPALO DIEKAOOH C2H5OH HCOOH HCOCO2H GLYOX ETHENO3O2 HYPERACET $\mathrm{CH} 3 \mathrm{O} 2 \mathrm{HCHO} \mathrm{CO} 3 \mathrm{C} 54 \mathrm{CO} 3 \mathrm{MEKCOH} \mathrm{CHOMOHCO} 3 \mathrm{H} \mathrm{CH} 3 \mathrm{OH}$ MACROHOOH HOC $3 \mathrm{H} 6 \mathrm{CO} 3$ NPROPOL C5CO234O CO3C4CO3H HYPROPO2 CO23C5 NO2 NO3 C4PAN3 MACO3 CO23C54O2 N2O5 H2O2 IPROPOLPER NC4H9O HO1C4NO3 HOC $3 \mathrm{H} 6 \mathrm{OH} \mathrm{HOCO} 3 \mathrm{C} 5 \mathrm{OOH}$ CO3C4CO3 PENTACID HO1C3NO3 NBUTOLBNO3 C3PAN2 C2H5O NBUTOLANO3 HOIPRCO2H HOCH2CO3 MACROHNO3 PROPACID O C5PAN 16 CH $20 O G \mathrm{C} 5 \mathrm{CO} 2340 \mathrm{OH}$ HOC $2 \mathrm{H} 4 \mathrm{CO} 3 \mathrm{H}$ CH2OOA HO2C 4 OH BIACETO ACO3 PBN NC3H7O HO1C3O2 PROLNO3 HOCH $2 \mathrm{CO} 2 \mathrm{H}$ ACETOL CO23C4CO3H IC3H7O2 NBUTOLAO IPRHOCO3H H2 HOCO3C54O2 C66O BUTALO2H CHOMOHCO3 NOA C66O2 BIACETOOH DIEKBOOH HO1C4O2 CH3OOH HCOCH2O HCOCH2CO2H PRPAL2CO3H MEKCO2 EIPKBNO3 PERIBUACID C66OOH EIPKAO2 CO2C3CO3 PROPGLY IPROPOLO2H CHOMOHPAN PERPROACID EIPKBOOH MACROOH C5PAN9 IBUDIALPAN O1D HOC $2 \mathrm{H} 4 \mathrm{CO} 3 \mathrm{CO} 2 \mathrm{C} 3$ PAN CO3C $4 \mathrm{CHO}$ IC $3 \mathrm{H} 70 O \mathrm{H}$ ETHO2HNO3 CH3CHO HO2NO2 C2H5OOH IBUTACID CH3CHOHCHO C2H4 C6PAN6 CO23C4CHO EIPKBO NO NO3CH2CO3 NA BUTACID EIPKBOH PHAN NO3CH2CHO C4PAN5 HO1C $4 \mathrm{OOH} \mathrm{NC} 4 \mathrm{H} 90 \mathrm{OH} \mathrm{CH} 3 \mathrm{COCH} 3$ PIPN MACO2H EGLYOX CH3CO2H HO1C3OOH MGLYOO CO23C4CO3 PERPENACID CO NC3H7NO3 EIPK CO2C3CHO C23 54 CHO HOIPRCO3H CO23C54OOH HOC $3 \mathrm{H} 6 \mathrm{CO} 3 \mathrm{H}$ ACRPAN C4H9CO3 NO3CH2PAN EIPKBO2 MGLYOX HOCH2CH2O CO23C3CHO IC3H7O NBUTOLBO PROPALO2 NC3H7O2 $\mathrm{HOCH} 2 \mathrm{CO} 3 \mathrm{H} \mathrm{HOC} 4 \mathrm{H} 8 \mathrm{OH} \mathrm{CO} 23 \mathrm{C} 54 \mathrm{O} \mathrm{HCOCH} 2 \mathrm{CO} 3 \mathrm{H} \mathrm{C} 66 \mathrm{CO} \mathrm{CH} 2 \mathrm{OO} \mathrm{HO} 14 \mathrm{CO} 3 \mathrm{C} 5 \mathrm{HONO}$ EIPKAOH SA HO1CO34C5 NBUTOLBO2 HOCH2CHO C2H5CHO PROPOLNO3 C3H7CHO C2 $55 \mathrm{NO} 3$ PRPAL $2 \mathrm{CO} 2 \mathrm{H}$ NO3CH $2 \mathrm{CO} 3 \mathrm{H} \mathrm{C} 5 \mathrm{CO} 234 \mathrm{O} 2 \mathrm{HO} 2 \mathrm{C} 3 \mathrm{CO} 2 \mathrm{H} \mathrm{HCOCH} 2 \mathrm{OOH}$ $\mathrm{CO} 2 \mathrm{C} 3 \mathrm{CO} 3 \mathrm{H}$ C23C54CO3H HOC $3 \mathrm{H} 6 \mathrm{CHO}$ IPRHOCO3 PPN HOC $2 \mathrm{H} 4 \mathrm{CHO}$ HYPROPO $2 \mathrm{H}$ 


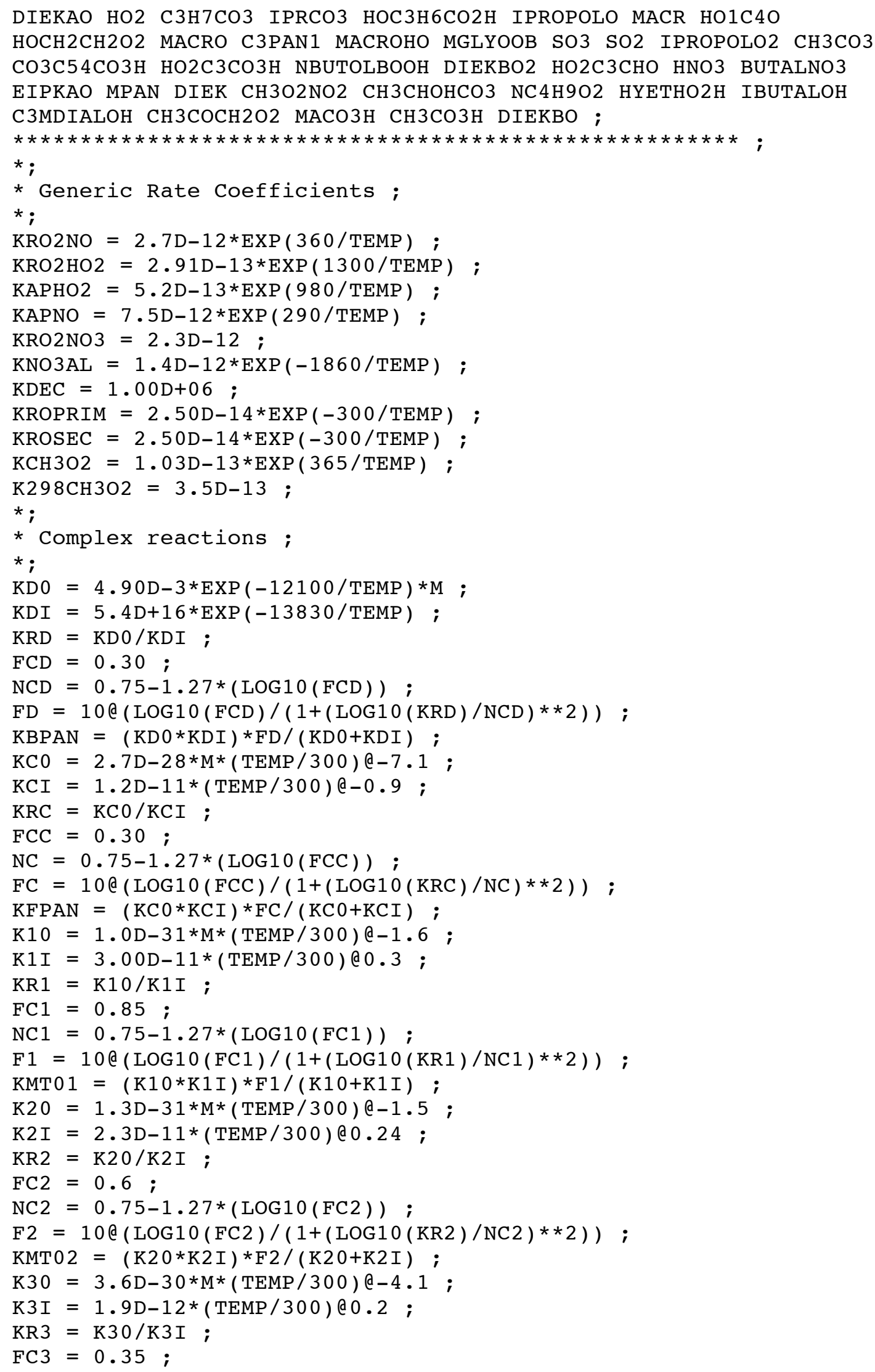




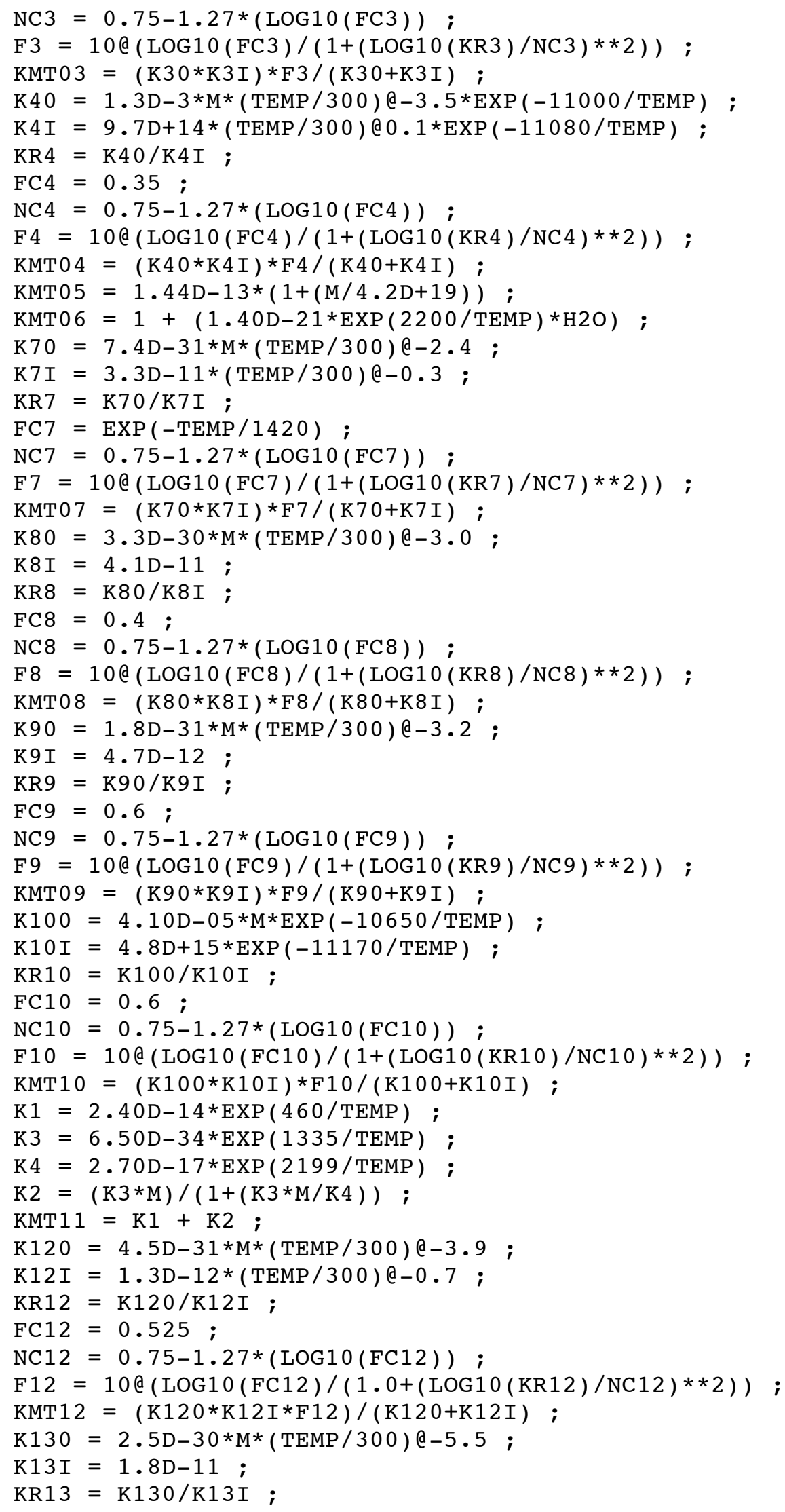




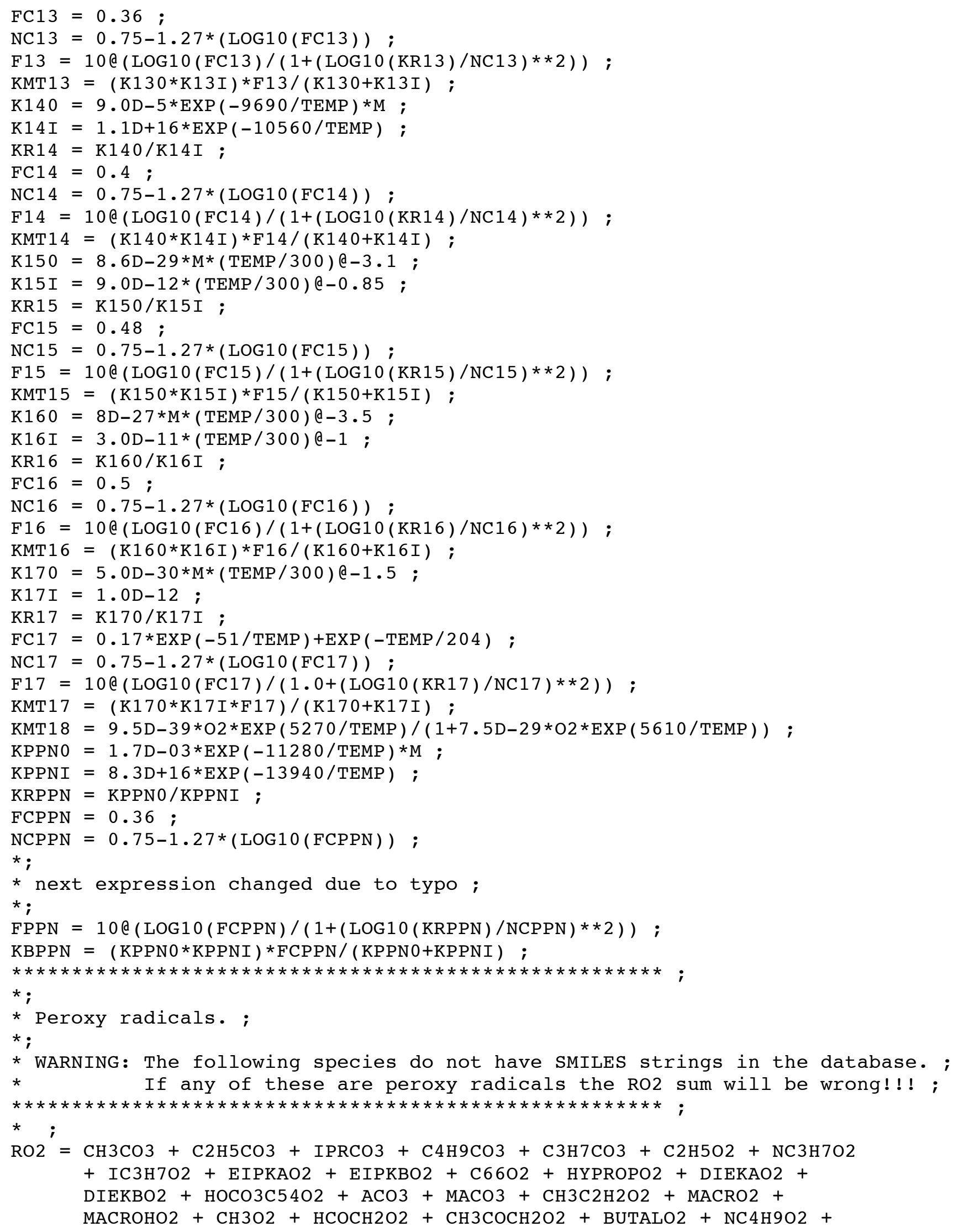




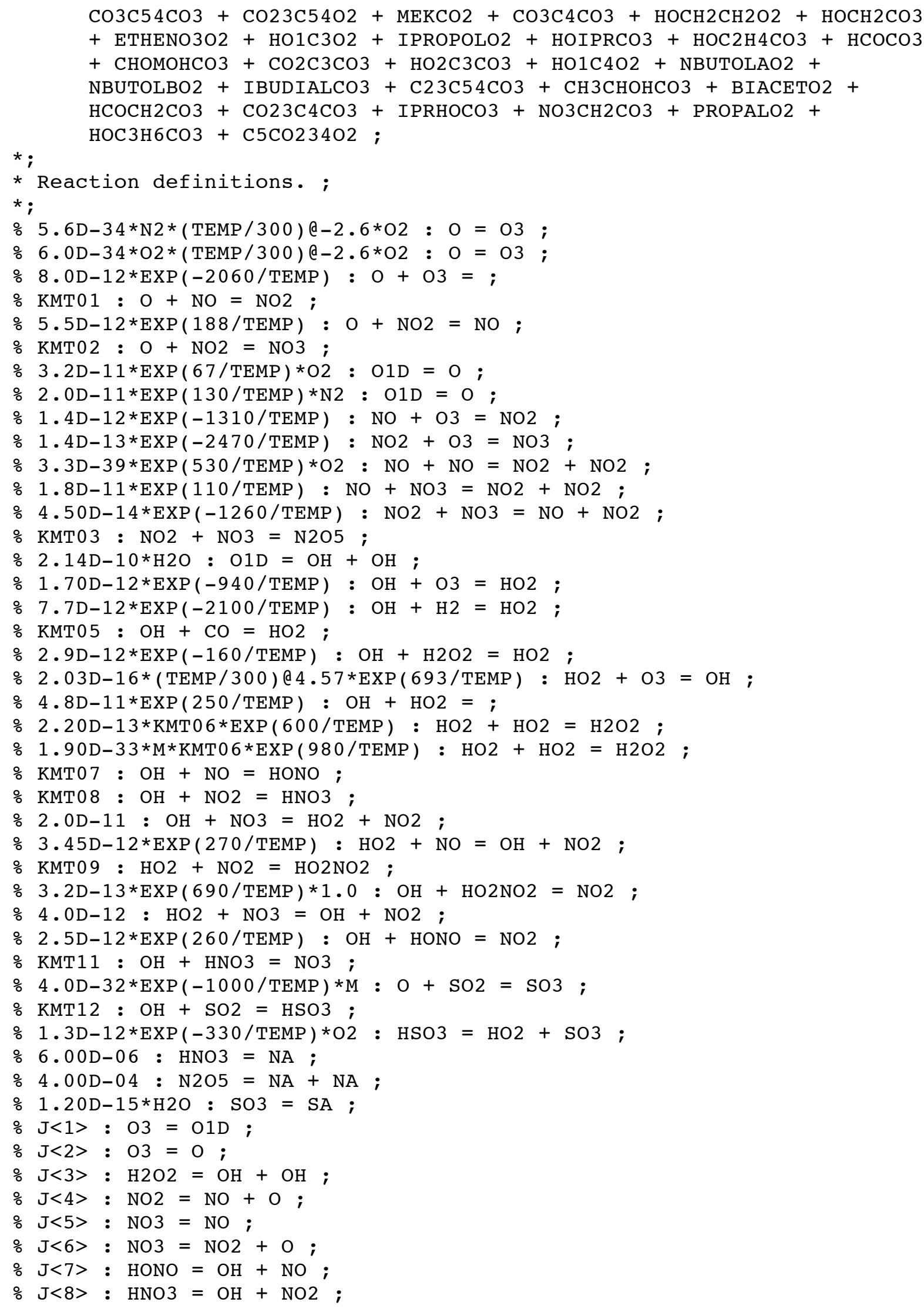




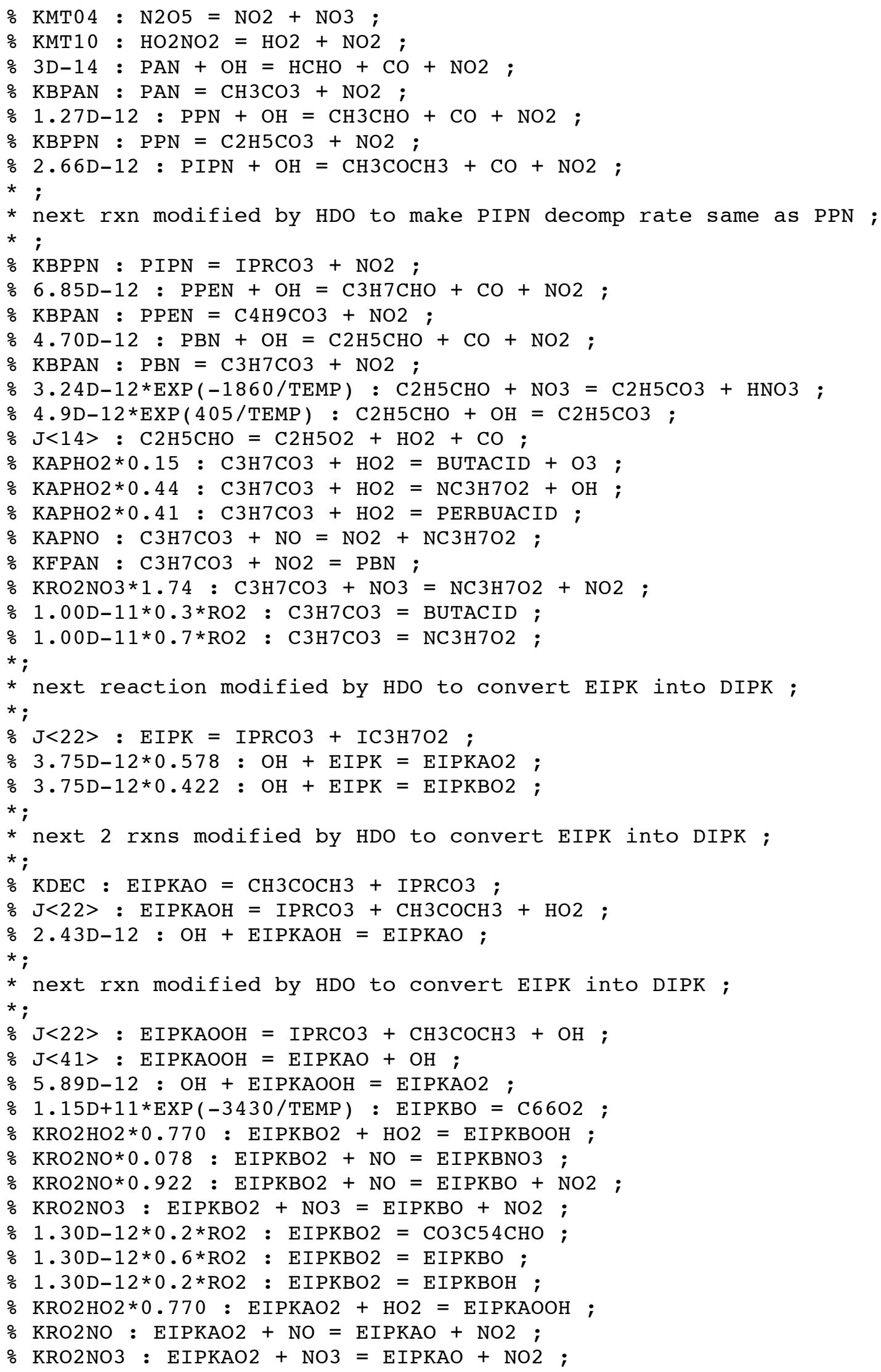




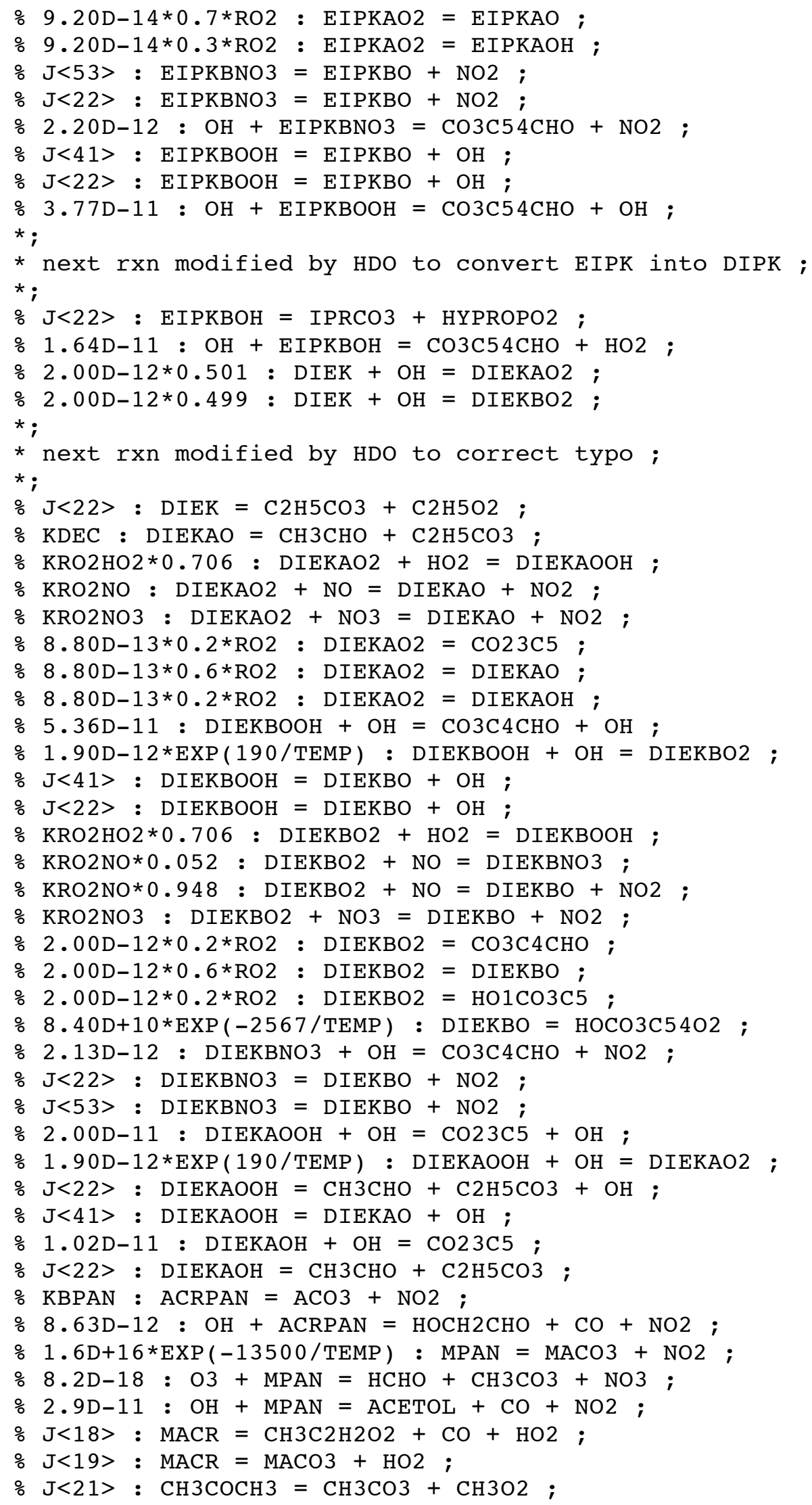




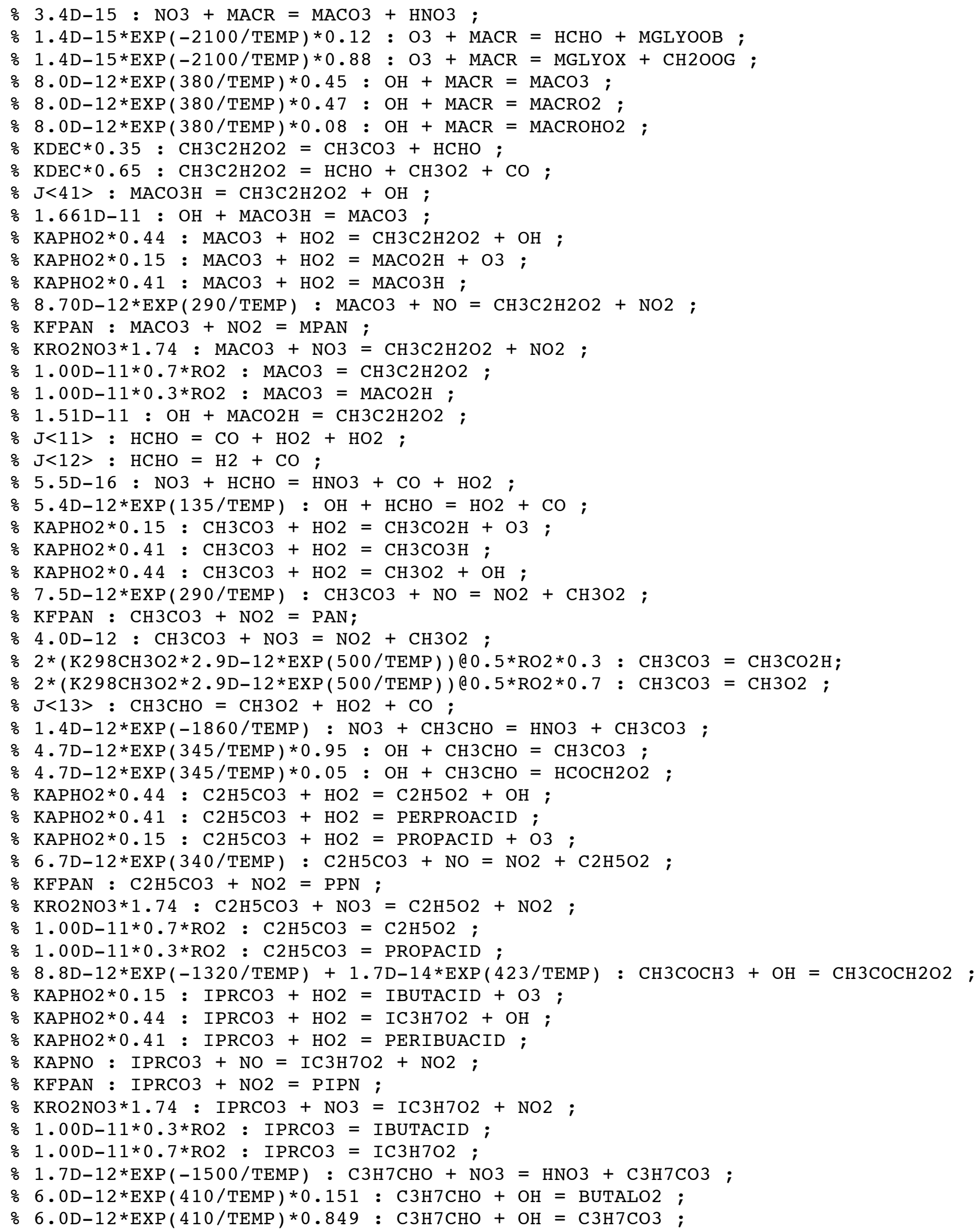




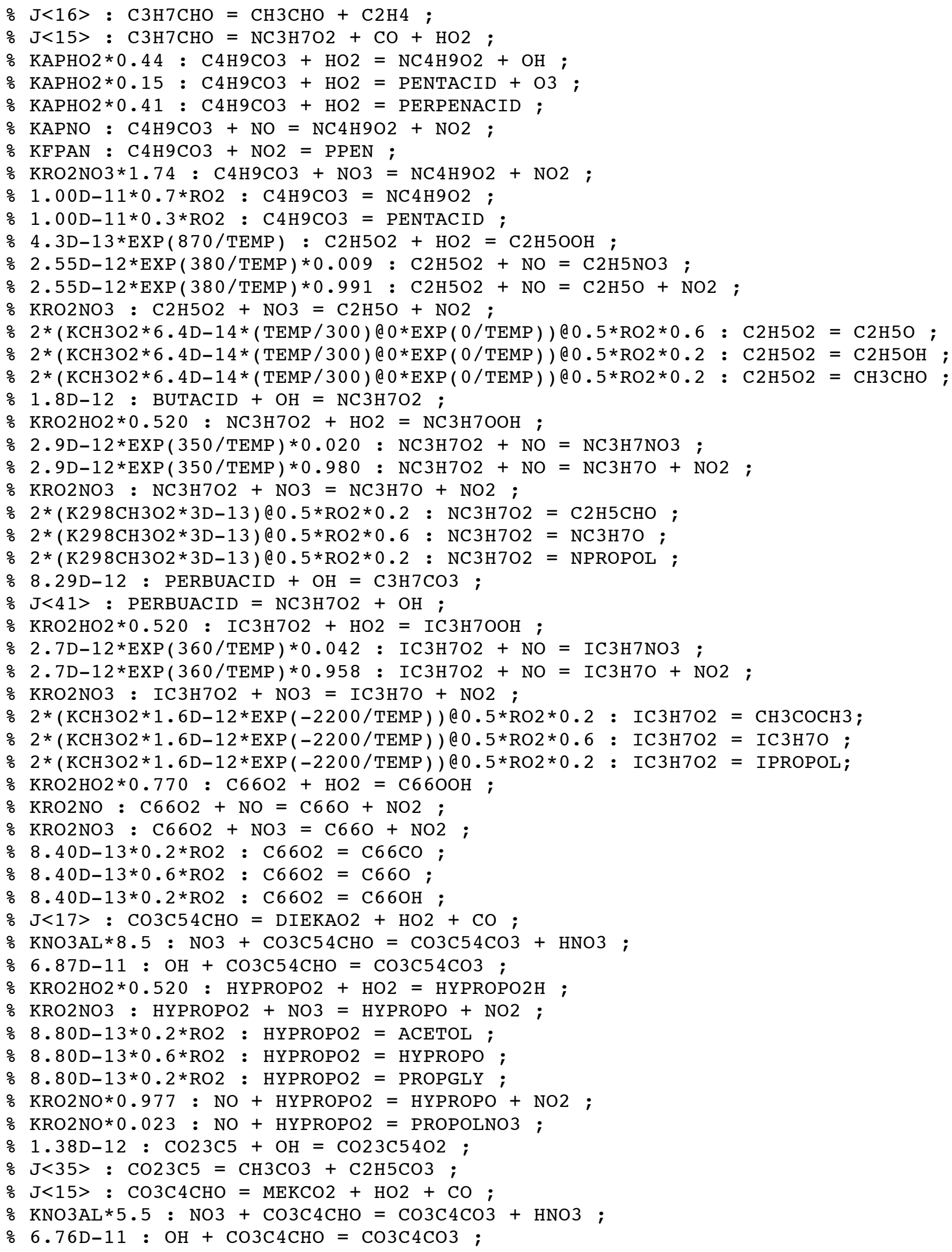




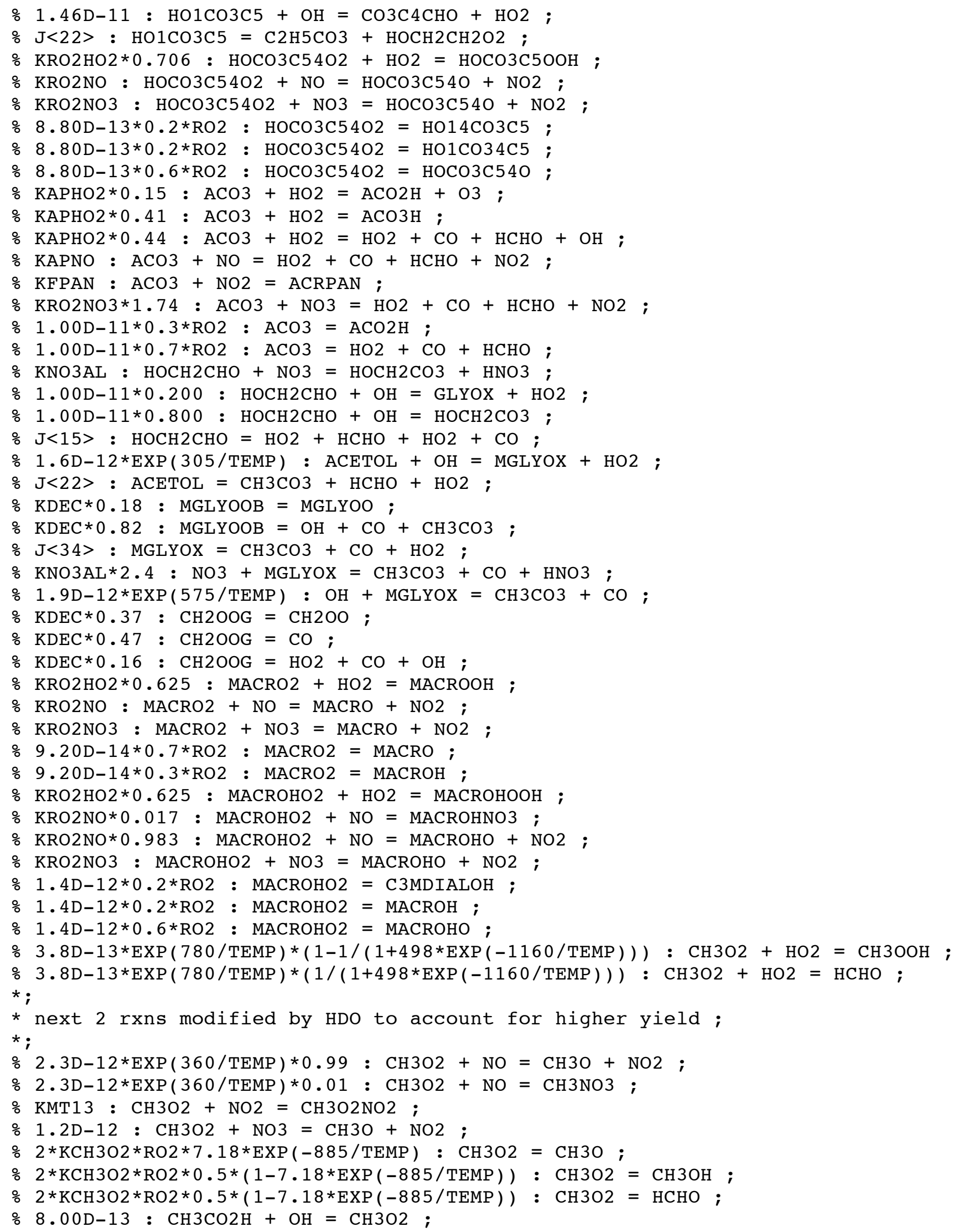




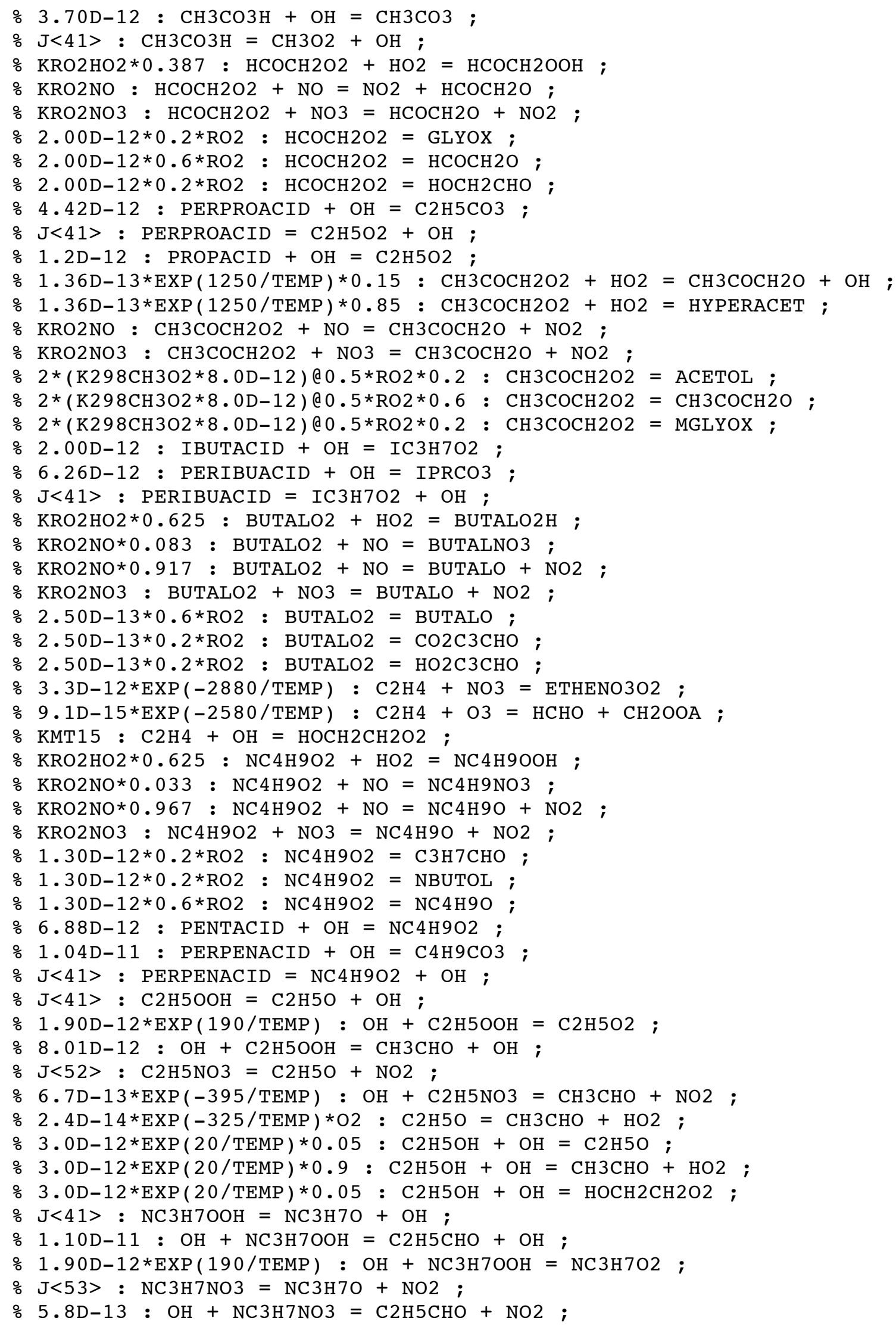




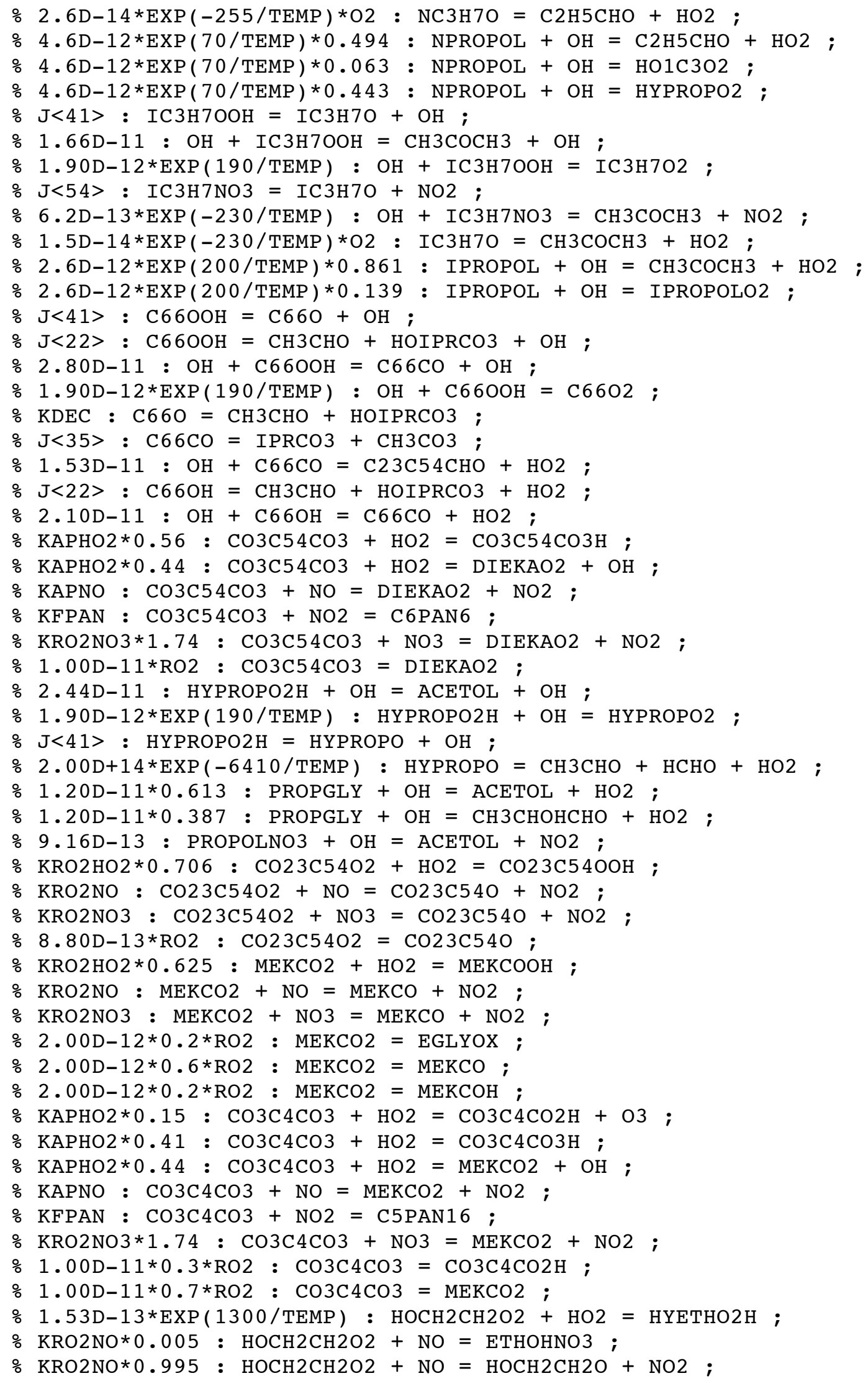




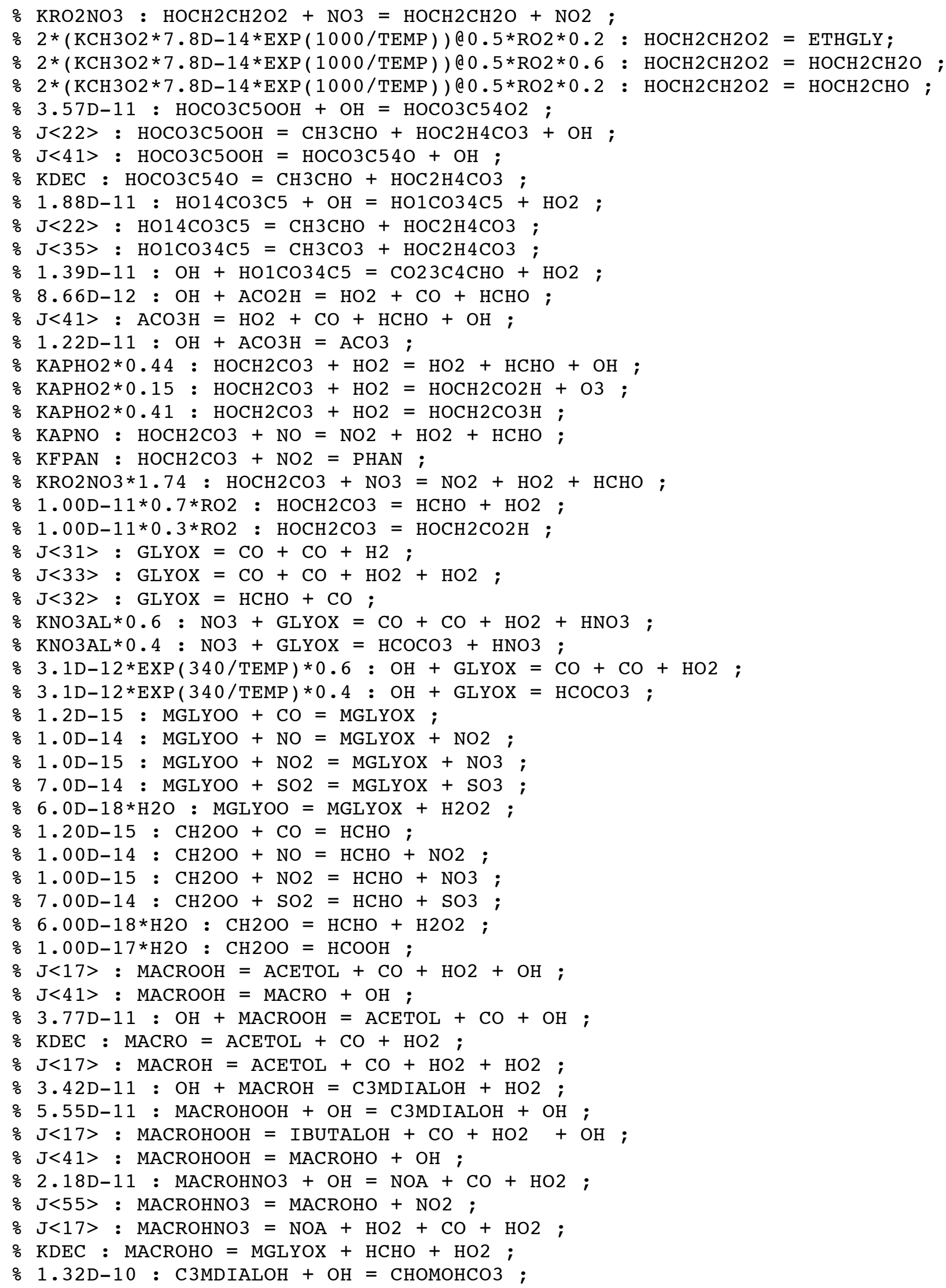




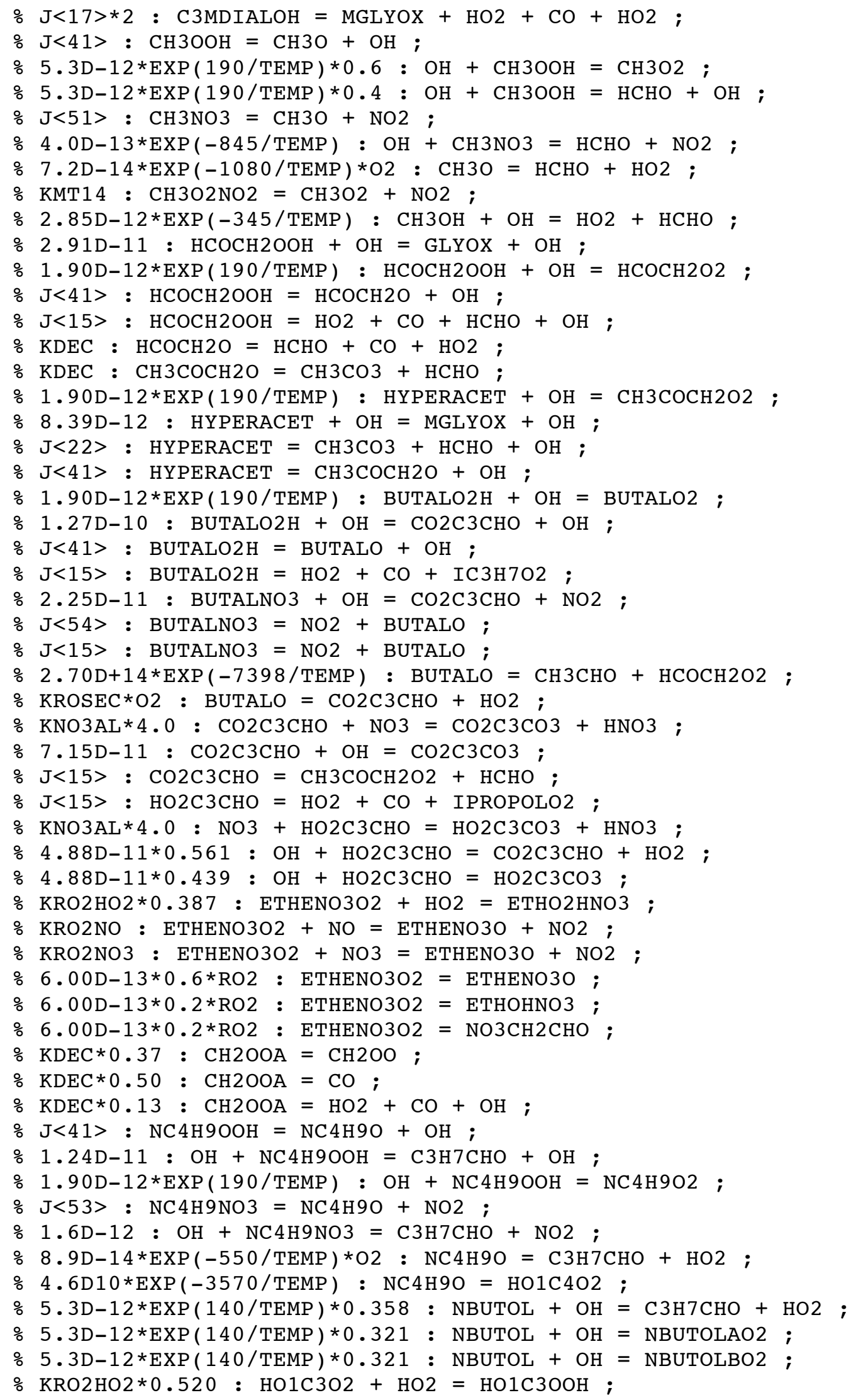




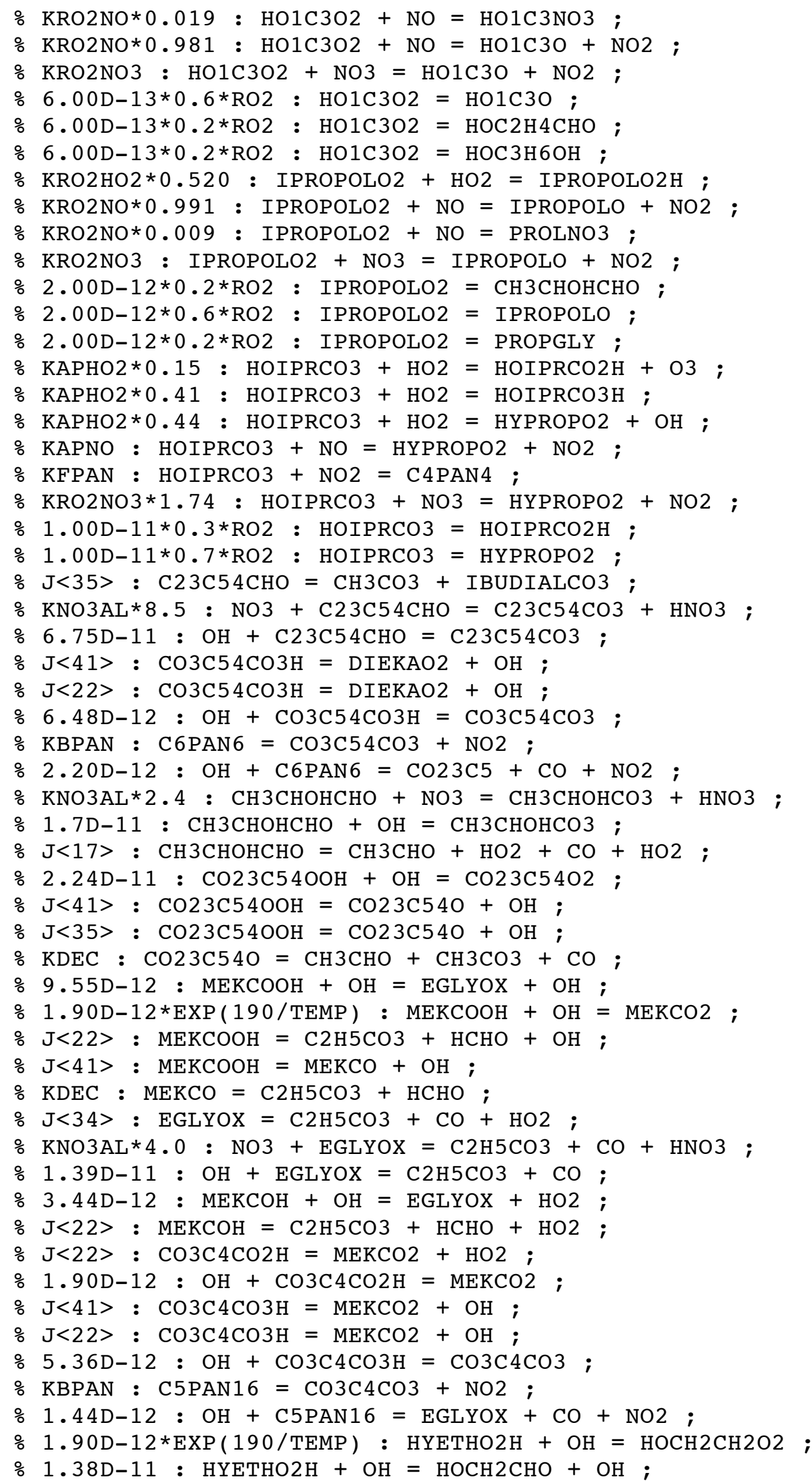




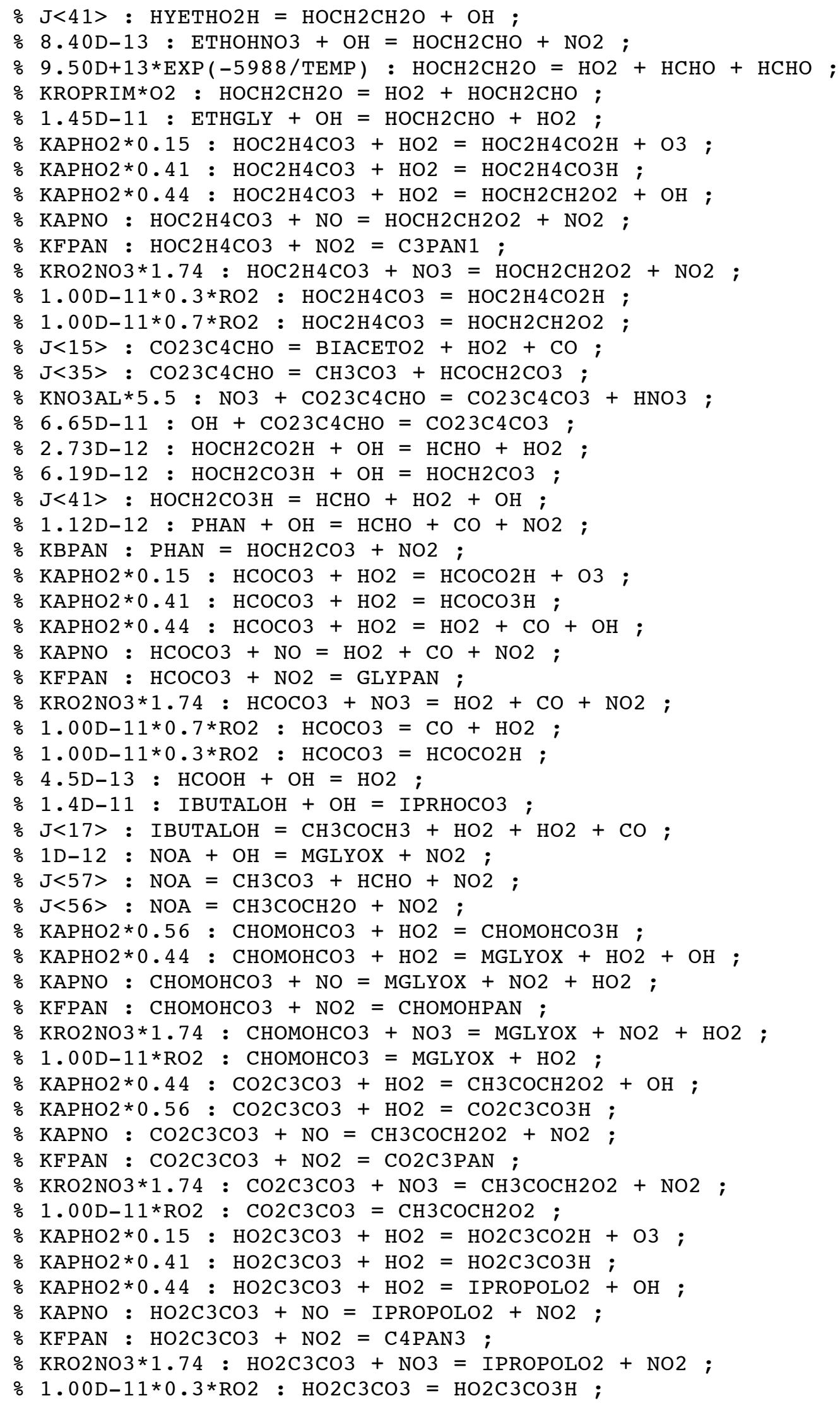




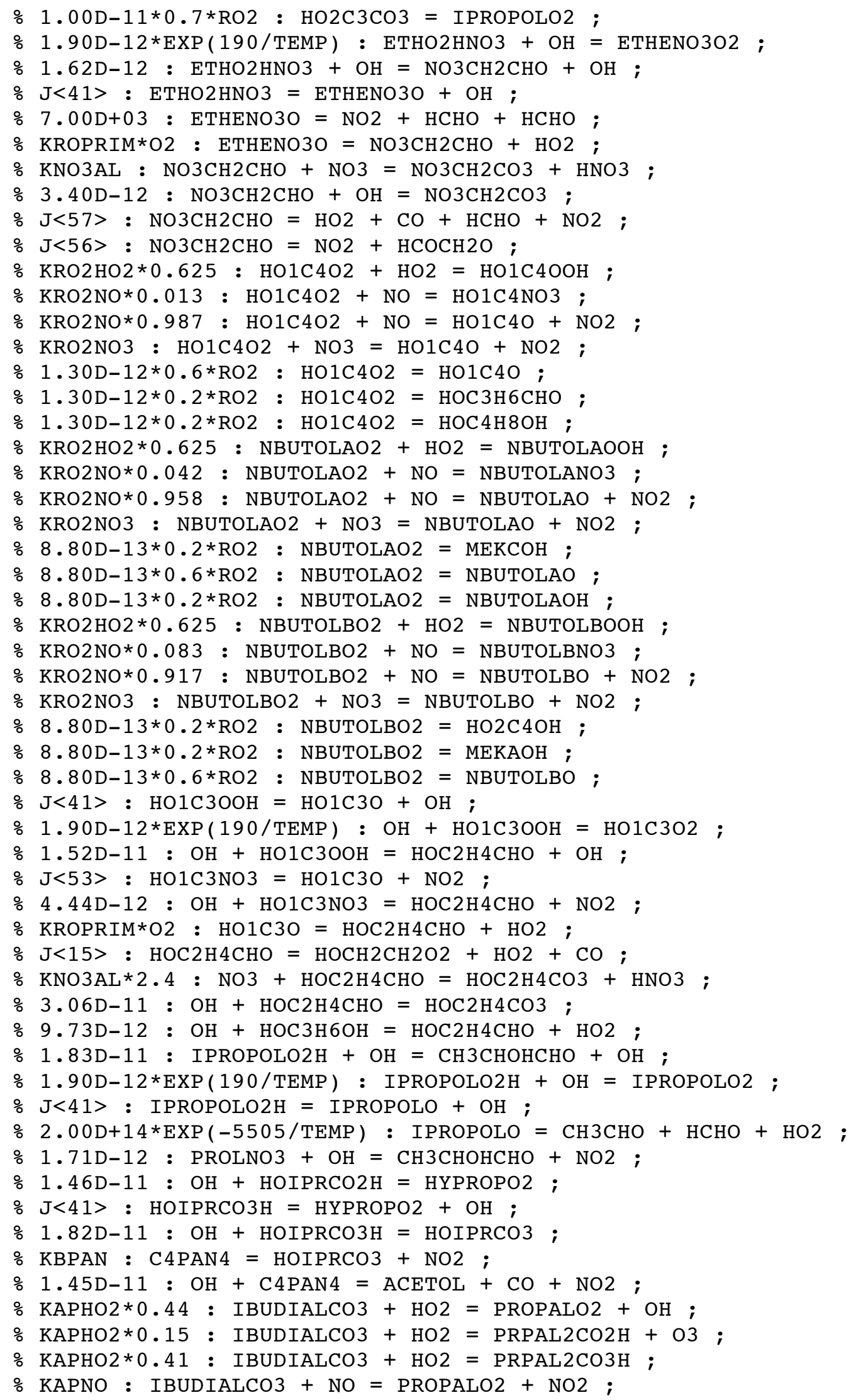




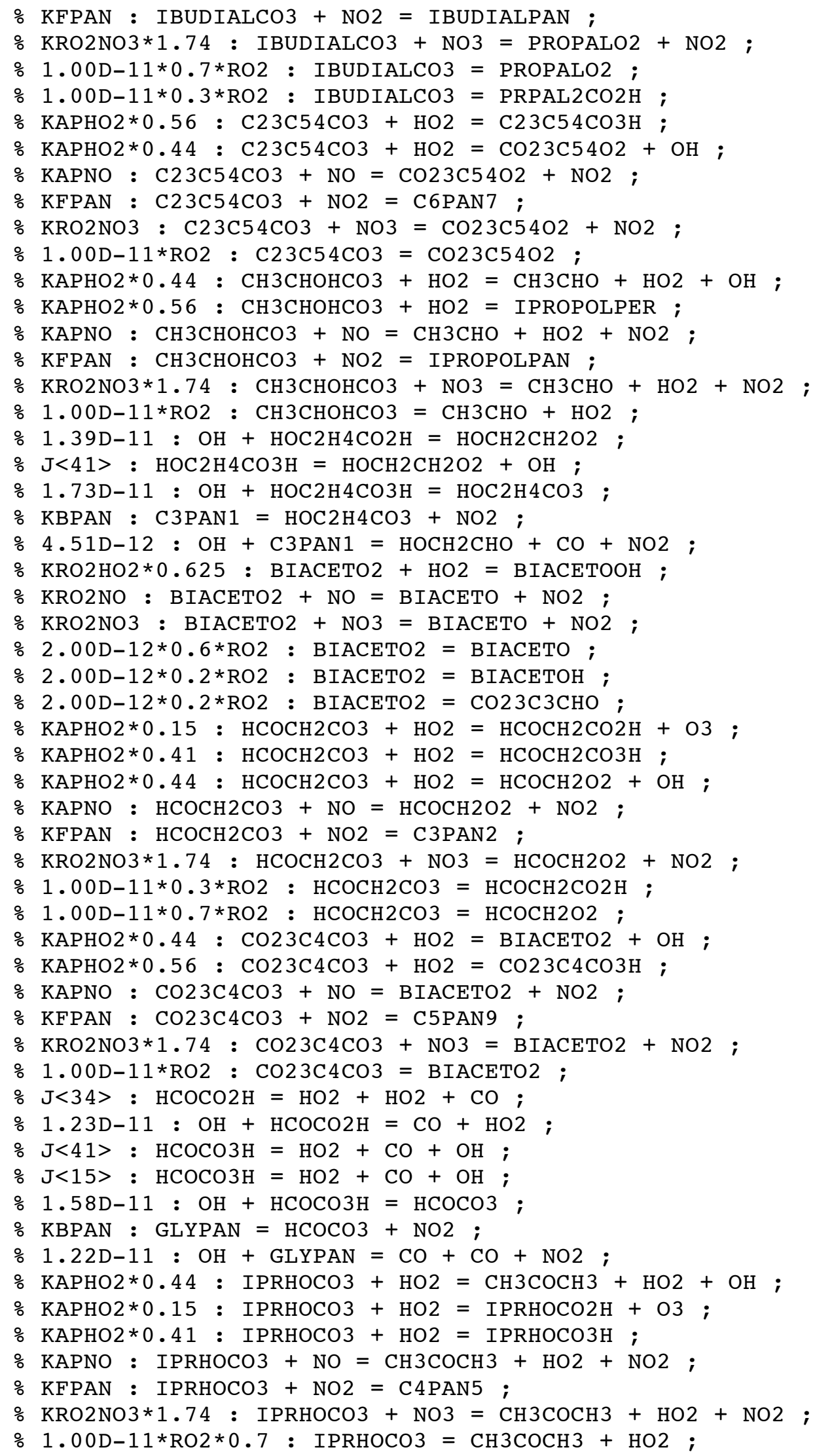




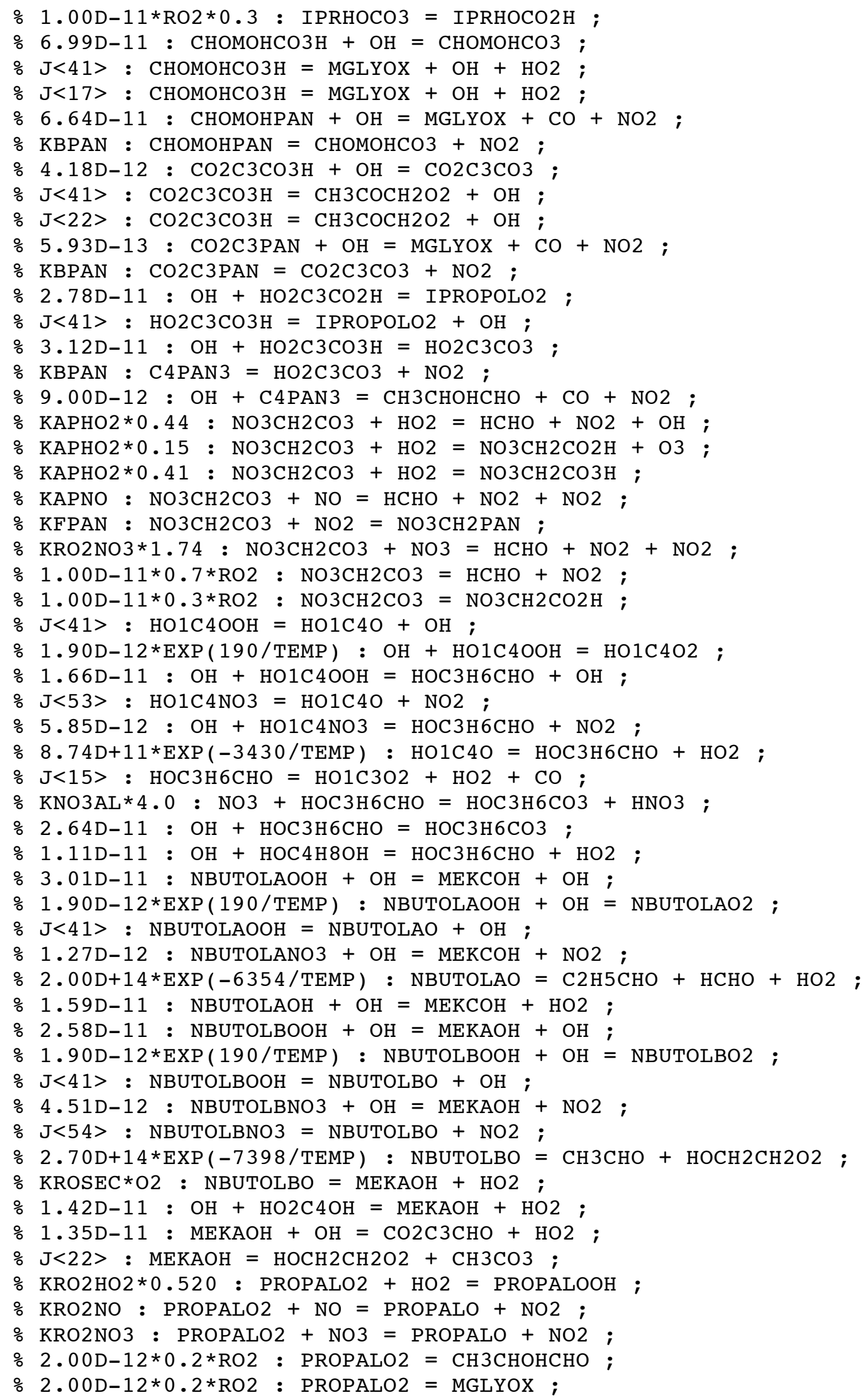




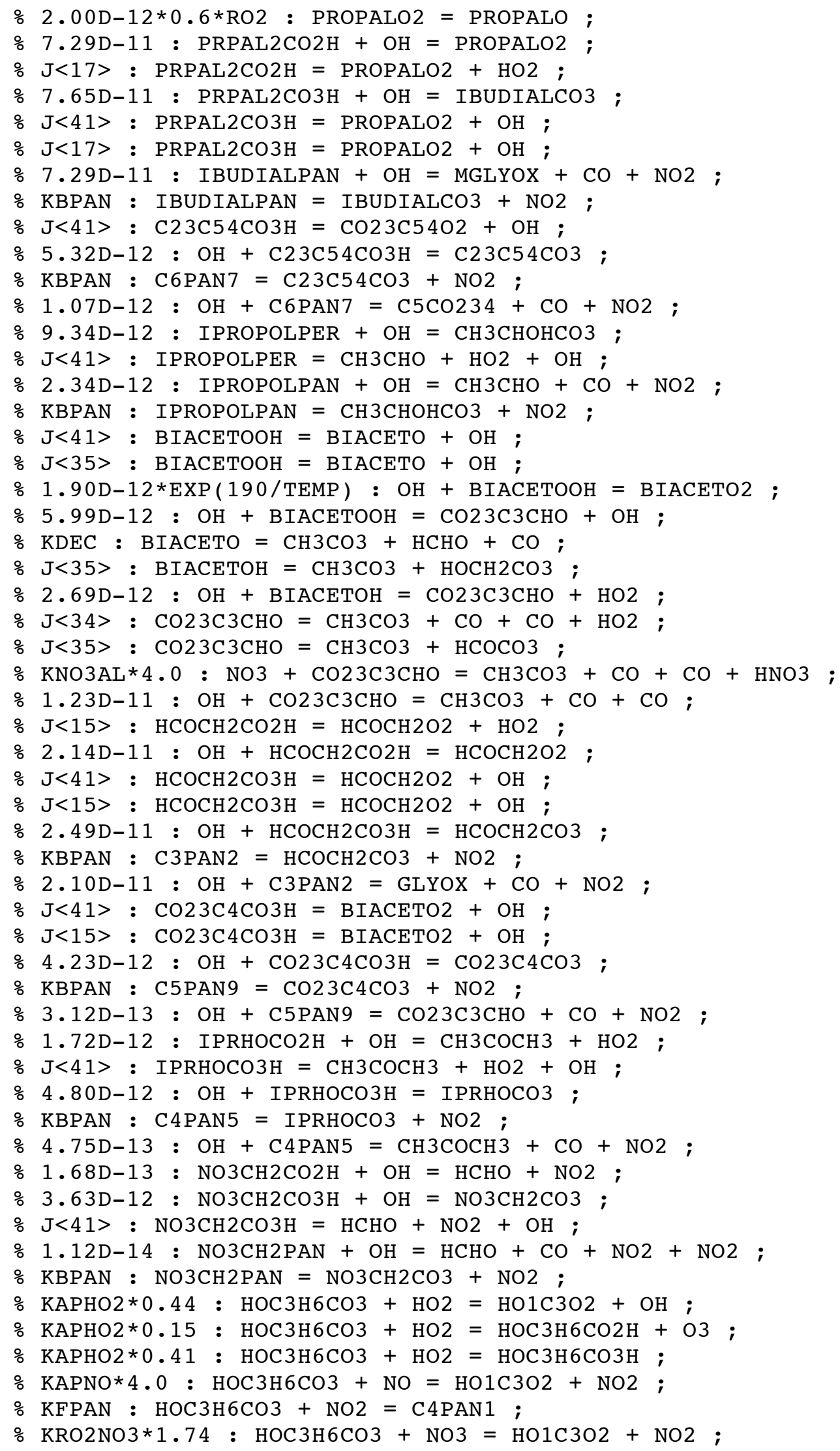




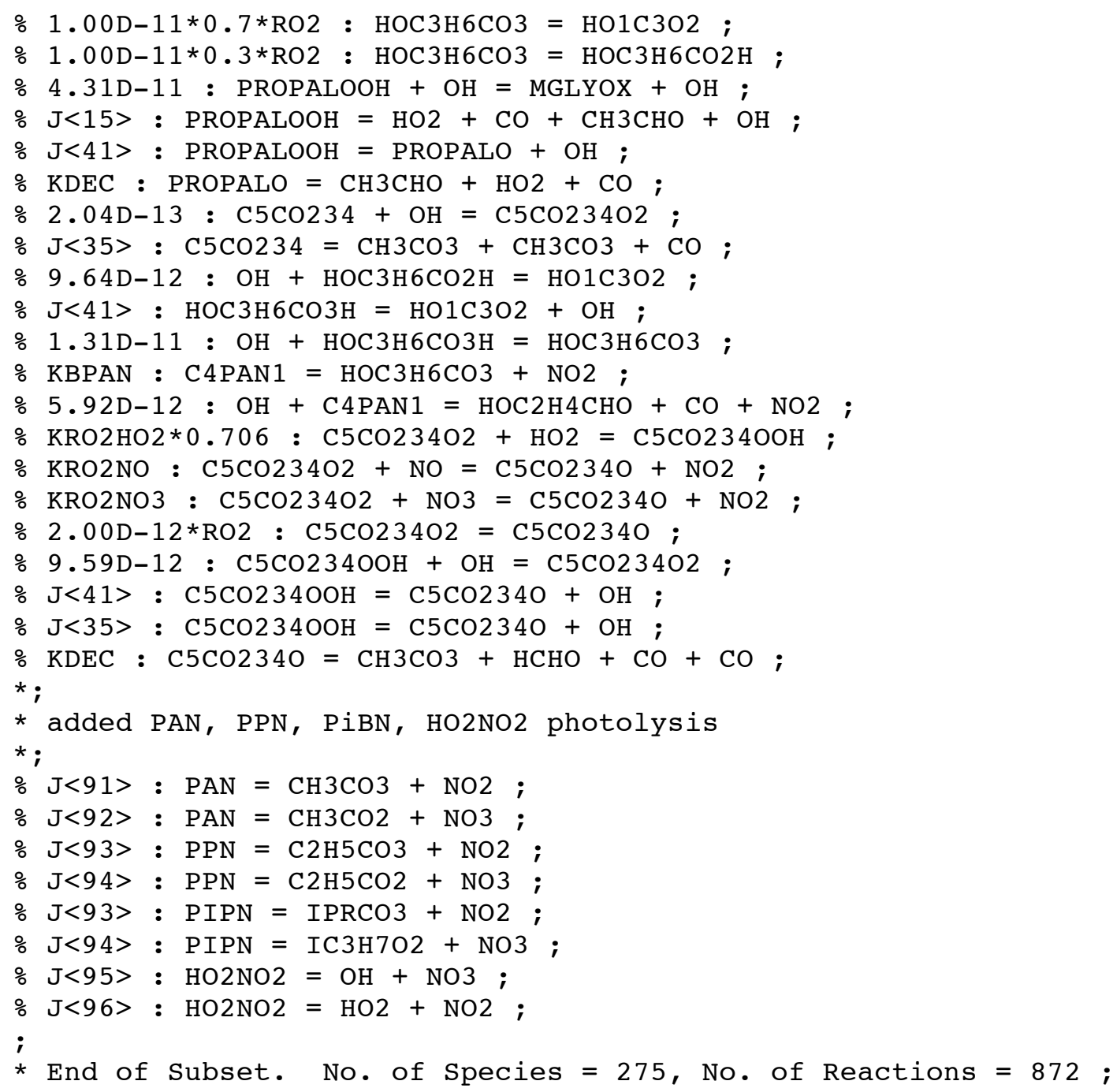

